\title{
Accelerated Simulation of Cell Biological Systems Using Heterogeneous Parallel Processing Platforms- A Survey
}

\author{
${ }^{1}$ Abhishek K, ${ }^{2}$ Sreenivasa.N, ${ }^{3}$ S. Balaji \\ ${ }^{1}$ Research Scholar-Jain University, Dept. of Computer Science \& Engg., Jyothy Institute of Technology, Tataguni, Off Kanakapura Road, \\ Bengaluru-560082, India, abhishek.mtech2012@gmail.com. \\ ${ }^{2}$ Research Scholar-Jain University, Dept. of Computer Science \& Engg., Nitte Meenakshi Institute of Technology, P.O. Box 6429, Yelahanka \\ Bengaluru-560064, India, meetcna@yahoo.co.in \\ ${ }^{3}$ Centre for Incubation, Innovation, Research and Consultancy, Jyothy Institute of Technology, Tataguni, Off Kanakapura Road, Bengaluru- \\ 560082, India, drsbalaji@gmail.com
}

\begin{abstract}
Simulation is a handy toolkit of scientists and engineers of all disciplines. Modeling and simulation helps in overall reduction of cost, increases the quality of products and systems, and documents and archives lessons learnt. This paper reviews modeling and simulation of cell biological systems in medical applications using heterogeneous parallel processing platforms. In heterogeneous computing, we use more than one kind of processor or core. This helps in achieving better performance not in terms of same kind of processing but with additional special tasks which require more computational power. The overall system gains efficiency due to the fact that there is offloading of computations to the Graphic Processing Unit (GPU).
\end{abstract}

Index Terms-Simulation, Heterogeneous Parallel Processing, Systems Biology, Cell Biological System, Modeling and Simulation

\section{INTRODUCTION}

$\mathrm{M}$ odeling and simulation refers to the representation of a system, or an entity using models and processes as the basis for simulations. Modeling and simulation basically aids in getting information about how something will behave without actually testing it in real life. The major task involved in systems biology is modeling. This can be achieved by using efficient algorithms, data structures along with various visualization and communication tools [1]. Modeling and simulation in systems biology helps in analyzing and visualizing the neural networks, metabolism, cellular subsystems and so on. These, in turn, help in understanding the core activities in the biological system and also know the various processes involved.

Modeling and simulation is the use of various models which might include physical or mathematical to implement a problem. Modeling and simulation helps in understanding system behavior without actually implementing in real world.

Hence it is useful to study system by subjecting it to various parameters which it might come under in real.This paper discusses about the modeling and simulationusing heterogeneous parallel processing in systems biology research. Simulation in systems biology is amenable for parallel processing since the biological process can be conveniently split into parts and attached to different processors in the same computer. Parallel processing speeds up complex computations of simulations in systems biology. Parallel computers are classified according to the hardware level parallelism. The multi-core and multi-processor computers have processing cores which have the capability to execute high throughput streams (the threads appear as streams as more and more threads are spawned dynamically) in parallel. Massively parallel processors also use multiple processing cores to work on the same task improving the overall processing speed.

Computational requirements are ever increasing in the areas of imaging, scattered databases, simulations, technical prediction etc. Sequential architectures have reached their physical limit. The technology of parallel processing with significant progress in research and development of tools and environment is mature now and can be exploited commercially. Developments in networking technology is also paving way for heterogeneous computing [1-2].

\section{Simulation OF BiOlOGiCAL SyStEMS}

Systems biology and mathematical biology along with computational biology aim to develop and use algorithms, data structures and other tools to model biological systems. The visualization and communication tools help in visualizing the model on a computer with the help of modeling rules given by the modeling tool. Modeling activities related to biological systems include:

- Cellular model building

- Multi-cellular organism simulation

- Construction of human biological systems

- Modeling of infectious disease

The cell has always been considered as a system since it comprises of numerous biological interconnected mechanisms. Cell biologists are interested in studying more features about the internal mechanisms which help in knowing more about the whole biological process. Systems biology helps in understanding of how information is transmitted and 
interpreted by the cell [5] [6]. The properties of the cell and interactions can be considered as a system consisting of logical gates and other combinations to analyze it. It is important to know the spatial and structural features of a cell to know more about the emergent properties. Merging technology and computation helps in revealing such emergent properties of a cell.

Systems cell biology involves study of those properties of a cell which emerge as a whole rather than individual property. This property is called as emergent property. Quantitative and comprehensive methods can be employed to study such emergent properties. Hence, systems biology employs modeling along with quantitative analysis which helps in understanding critical processes that are part of cellular organization and cell dynamics. Advancement in technology and computing helps in clearing the unresolved issues in cellular mechanisms. Emergent properties indicate that the whole is bigger than sum of its portions; essentially the whole property has significant impact than the individual property.

\section{Heterogeneous PARALlel Processing}

In HPP, the data processing requires effective use of multiple Central Processing Units (CPUs) with General Purpose Graphics Processing Units (GPGPUs) for computation. The combination of CPUs and GPGPUs increases the speed of processing. The idea behind HPP is to offload part of CPU load to the GPGPU which will improve the performance in terms of speed. The challenges of programming Graphics Processing Units (GPUs) include alteration of the code for parallel processing, dividing the code into kernel code to be executed on the GPU and the code to be executed on the CPU, allocation and synchronization of CPU and GPU memory, etc. GPU-accelerated computing uses a GPU with the CPU to quicken the scientific applications and simulations. GPUs contain many cores which are capable of performing instructions in parallel. These cores are made up of millions of transistors which are capable of efficiently performing floating point computations. By using the computational power of GPUs one can significantly speed up compute bound and graphics intensive simulations [18].

Graphic processing units were originally used to quicken memory intensive processing involved in graphics interpretations like texturing, mapping and rendering. Simulation of scientific systems involves memory intensive procedures along with floating point calculations. Using GPUs we can quicken these processes [17].

\section{Heterogeneous Parallel Processing In BiologicAl APPLICATIONS}

The Section is divided into four subsections each highlighting the use of heterogeneous parallel processing for simulation of specific aspects of a cell biological system. Subsection 4.1 presents various studies carried out in accelerating complex interactions in the cell biological systems. Subsection 4.2 discusses HPP acceleration of gene expression connectivity mapping. Subsection 4.3 deals with virtual screening for drug discovery and Subsection 4.4 discusses about spiking neural networks.

\section{A. Accelerating Complex Interactions in Cell Biological Systems}

Cell biological systems often consist of complex interactions which involve many cells and its biotic and abiotic background. Systems biology, also called as computational biology, aims in predicting and manipulating the biological system using mathematical models and computer simulations. One popular approach for building biological system models is discrete agent based modeling [32]. The high computational complexity forces system biologists adapt GPU based computing. High Performance Computing (HPC) has the potential to address this issue but requires significant effort and time to develop parallel processing code.

1) Drug Delivery Systems:

Drug delivery involves techniques, systems and formulations for carrying preferred complex structured medicine as needed to cautiously accomplish the therapeutic result. It involves targeting the compound in the body or might involve simplifying pharmacokinetics. In all the cases, it is timed together with capacity and period of drug. Drug delivery is part of dosage and route of administration and also includes medical devices or drug device mixture products.

Delivering required drug compound into specific organs or tissue is a critical research issue. Hence, drug delivery is a multidisciplinary scientific approach. Antibody-Mediated Drug Delivery System (ADDS) [1] is developing as a promising method for treating cancer patients. The technology makes use of the antibodies which are large in number and is provided to stick to the receptors of the sick cells known as antigens. The Molecular Communication (MC) [2] being the core part of the atom provides support in constructing ADDS. ADDS uses point-to-point response for infusion of medicine into cells. Breakdown of medicine from the cells is numerically calculated based on the cell geometry that counters these acting agents. The kind of accuracy received from MC depends upon the limited module restorations. The MC paradigm is used to carry the data through the particles [4].

ADDS contemplates the exclusive properties of the antibodies and the possibility of addressing the drawbacks in the physiologically-based pharmacokinetics prototypes are being used for molecular communication in ADDS. These frameworks do not assist in providing the dimensional accuracy to measure the competence in ADDS [1].

The simulation methods are designed and advanced to show the communication between cells in the biological systems. The researchers in molecular communication primarily focus on the interaction between the biological cells. They also focus on how, by reengineering, they can produce new communication methods for the biological systems. The simulation method NanoNS [31] has been designed to represent molecular communication through diffusion processes. The simulation method N3Sim [31] is also used for 
the same purpose as that of NanoNS simulator. There are no tools being used for evaluation of molecular communiqué networks. The main experiment here is to bring many tools into a single platform and aid in evaluating various designs to molecular communication networks. Molecular communication is said to be used in the Nano machines [4]. How the communication takes place between the nano particles in the nanomachines are to be seen. The simulation platform BiNS2 [31] helps in simulating nano particles communication.

2) High-throughput Biological Cell Classification:

The classification of the biological cells which features realtime compression of the optical data uses telecommunication devices to produce, collect and then examine the collected data at high computational speeds using GPUs. The method used for capturing the real-time signals is time-stretch dispersed method of the Fourier transform [7-8], [12-15]. It lets the capturing of optical spectra in a stretch with millions of frames per second speed leading to the rogue wave detection [7] and collaboration to track the conversion of the signal from analog to digital [8]. A camera is used in the telescope known as STEAM [9-11] that detects the breast cancer cells in blood with great sensitivity [18-23]. Another technique called FIRE [12] has very high capturing speed with higher magnitude as the present gold standard in great-speed fluorescence imaging [25]. Even with the most advanced computers, the biggest problem faced is producing real-time data of the order of one tera bits per second [26-30]. The compression method for faster real-time operation uses a completely different method to get similar functional compressive sensing [22].

The real-time optical data compression requires high performance computing to attain the output in real time. To get high concert computing at the data rate of $970 \mathrm{Mbps}$, a panchromatic band and multi-spectrum band are used in the Remote Sensing Instrument (RSI). There are 3 Xilinx Vertex and 5 Field Programmable Gate Arrays (FPGAs) with the external memory which are used to perform optical data compression in real time. To accomplish high computational performance - parallel and concurrent handling approaches are used in the process [33].

\section{B. Gene Expression Connectivity Mapping Through HPP Acceleration}

In this sub-section, we discuss the HPP acceleration of gene expression connectivity mapping. There exists significant interest in gene monitoring networks and gene-regulatory memories by electrical-equivalent modeling, simulation and parameter documentation. In gene communication, the evidence from a gene is recycled in synthesis of an efficient gene product. The resulting products are typically proteins except in non-protein coding where the product is a efficient RNA [24]. The gene expression procedure is used by all types of life like bacteria and achaea and includes multicellular organisms to generate macromolecular machinery for life.

\section{1) Reconstruction of Large-Scale Gene Regulatory Networks}

Gene regulatory system is a group of controllers that interrelate with additional elements in the cell to oversee gene communication stages of the RNA and proteins [11]. The communications of cells are direct or indirect. Commonly, mRNA molecule emerges to make a protein or set of proteins. Gene regulatory networks give enormous information regarding the interaction of different molecules in a biological system [17]. The main challenge is to build a large-scale gene regulatory system. The procedure includes a lot of information. GRN uses Bayesian model that plays an essential role in determining altered biological complications, where it can identify the transcription factors of specific illness indicator genes [18] and is utilized to find out the information in the developmental processes that influence the improvement of some disease conditions [19].

\section{2) Gene-Regulatory Recollections: Electrical Equivalent Modeling, Reproduction and Parameter ID}

The progress made in gene-regulatory memory circuits [23] has made breakthrough in finding out the information that has been stored and has enabled in new applications. These applications and generations give quantitative examination and findings of the practices and components of the frameworks that have been inherited. The reactions are mapped in the process of electrically-similar models and the framework that is being exceeded by artificial SPICE-like circuit diversion environment [11]. The researchers address the most important problem related to showing framework model parameters by adding to a multiplication driven Bayesian structure for recognizing various parameters. To make sure the trustworthy ID of some of the crucial system properties, the researchers have proposed a two-stage structure that defends parameter unmistakable confirmation philosophy [28]. Initial step collects bi-strength, the most basic characteristic of a memory methods and the subsequent stage is regulating to recognize dynamic belongings of target while protecting up to the observed bi-security.

\section{Virtual Screening for Drug Delivery}

In this section, we discuss about the parallel computations for medical applications in virtual screening for drug discovery. This basically involves finding protein-ligand affinity. Affinity indicates how strongly the ligand gets bonded to the protein. More the affinity more likely the desirable properties will be exhibited. The process involves finding a location where the ligand bonding is more. This is highly computationally intensive task. The reason behind such intensive calculations is because of the 3-D structure of protein which must be explored. This results in more floating point calculations. Also, visualization tools require more frame rate. This cannot be achieved using a normal CPU. This task can be accomplished using GPU in conjunction with normal CPU. In this process, the researchers investigate where a ligand can be tied to specific protein [24-25]. Some methods 
like ADAM, DOCK first create a ligand adaptation and then docking is done.

Binding a ligand to a protein involves two parts. The first is to discover the conformational galaxy of the ligand. Next is to record the function which can be recycled to assess the exposed binding modes which are known as poses. A scoring function must allocate the finest mark to those positions which matches more with the crystal structure. The prediction of accurate binding mode is very crucial which forms the first step since it is the major part of the docking process. In the second phase, poor binders or non-binders should be identified and active compounds should be assigned with higher scores [21]. Modeling and simulation in bioinformatics mainly concentrates on simulation of biological systems which helps to analyze a biochemical reaction or may be how stimulus results in various subsequent triggers [26]. This information might be helpful to get a closer view of the process and hence helps in discovering any deviations in the normal process.

Figure 1 shows time taken to dock protein to ligand [10] in single core and multi core systems. The current drug discovery procedure is centered on discrete targets and streamlined measures. When we consider the objective, certain improvement exercises get to be less demanding, but on the other hand is a critical drawback. The single-target methodology is less effective. The single target based approach likewise restricts the capacity of scientists to distinguish creative targets and/or components of activity by constraining druggable space [27] to perceived targets and modalities.

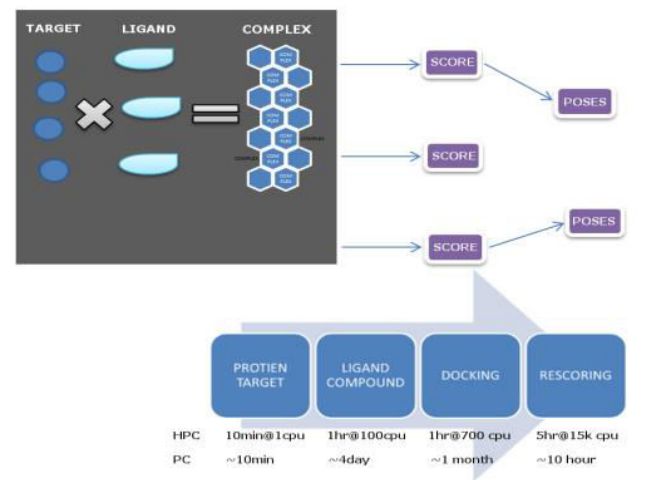

Fig. 1 Comparison between Single Core and Multi Core in case Protein to Ligand Docking Time

\section{Spiking Neural Networks}

Spiking Neural Networks (SNNs) fall into third generation of neural network simulations, updating the steps of reality in a neural model. In addition to neuronal and synaptic step, spiking neural networks also integrates the idea of time into the functional model. The knowledge is that neurons in SNNs do not fire at every transmission phase, but somewhat fire merely when membrane potential, fundamental feature of neuron associated to its membrane charge, touches a charge [13]. If neuron excites, it produces sign which moves to another neuron which in turn, updates or reduces their capacities in accordance with this sign. Simulation of spiking neural network is unique to the interesting computational responsibilities because of its numerical complication and simulation time. Aimed at actual time simulation, a huge number of mathematical calculations are to be extremely parallelized. The basic neural network is demonstrated in Figure 2. The neurons are designated by the mark $\mathrm{Nj}$ and the pre-synaptic relations $\mathrm{Xi}$ of a neuron are shown as minor circles through the weight Wi [18].

The synaptic weight calculation formula is
Synaptic weight $[\mathrm{j}]=\sum_{1}^{n} X_{i} W_{i}$

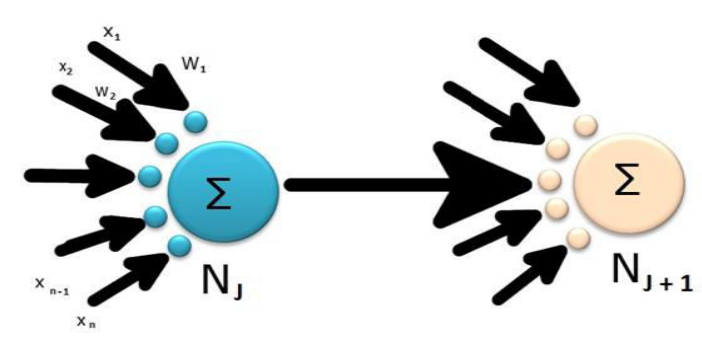

Fig. 2 Simplified Spiking Neural Network

In spiking neural networks, the present stimulation stage is usually measured to be the neuron's step with entering spikes moving this charge advanced step, and again firing or decomposing over a period. Different coding approaches occur for understanding the departing spike sequence as a realvalue numeral, either trusting on the regularity of spikes, or scheduling between spikes, to encrypt evidence [13]. This type of neural network can, in belief, be used for data manipulation requests in the same way as old-style artificial neural networks. SNNs can model the central nervous system of a computer-generated insect for looking for nutrition without the previous information of the atmosphere. Though, with their realistic properties, they are used to learn the process of biological neural paths. Beginning with assumption of the topology for biological neuronal paths and its purpose, electrophysiological footages of the paths can be related to the productivity of the analogous spiking artificial neural network replicated on processor, defining the credibility of the opening theory [29].

Artificial neural networks becomes nearer to biological samples resulting in promising emulating parts of biological nervous method to learn procedures which usually occur in the brain. However, they are not yet totally unanimously agreed upon by researchers. In specific, SNN simulators permit the study of the estimation realism in the study of procedures such as:

- Identification of how theoretical prototypes estimated biological procedures

- Learning of mental diseases by matching brain illnesses

- Challenging how medicines disturb the brain

The final choice of investigation relating to SNNs is to recognize in what way the human brain operates. Computers have continuously spent enormous resources to replicate biological neural networks. Nonetheless, they are not even close to imitate small processes that nature produced: Simulation of a collection of neural cells is a computationally 
daunting task. The computations for every component being simulated demand enormous calculations to be tremendously fast. The influence of brain is to branch from the massive parallelism of interrelated features. This is the basis of motivation for original schemes. Experts try to follow these features on the origin that these may signify the prospect of computer knowledge.

\section{1) The Event and Time Driven Hybrid Simulation of Spiking Neural Networks:}

Jesus et al [30] suggested spiking neural simulator incorporating the event driven and time driven calculation methods with parallel CPU-GPU systems. Time-driven model methods in old-style CPU designs precisely simulate limited spiking neural networks. Yet, they have disadvantages while simulating significant schemes. Also, event driven model approaches in CPUs and time driven model approaches in Graphic Processing Units (GPUs) can exceed CPU timedriven approaches under certain situations. Through this awareness in development, the researchers need to develop an event and time-driven SNN simulator appropriate to the hybrid CPU-GPU stage [15]. Their neural simulation can simulate bio-inspired SNNs enclosing dissimilar neural replicas. They describe simulator which incorporates dissimilar simulation approaches with dissimilar simulation methods; that is, time driven approaches in CPU and GPU besides event driven approaches in CPU create dissimilar treating stages in similar simulations.

As in [30] [17], an Event Driven Look Up Table (EDLUT) simulator can be allocated into three different sections: (i) neuronal dynamics, (ii) spike propagation and (iii) event queue management. Therefore, the calculation time could be allocated into t_neu_dynamics, t_spike prop, and t_queue_manag, respectively. EDLUT customizes numerous methods to recover the neuronal dynamic calculation. Therefore, this simulator achieves superior performance after the neural incorporation phase (i) conquers over others (ii) and (iii). Five phases are essential to appraise the neural state variables of every cluster of time driven neurons in GPU. They are:

1. Moving the supplementary update conductance path after CPU to GPU.

2. Apprising neural state variables through the fresh entering conductance standards. The GPU allocates true in its matching Boolean vector location or untrue otherwise.

3. Moving the Boolean vector after the GPU to CPU.

4. Rearranging the supplementary increasing conductance path.

5. Producing the productivity of spikes in CPU from Boolean vector

Parallelizing time driven approaches on CPU and GPU together recovers the routine of simulator though retaining its elasticity and correctness. The parallelization process will permit the usage of additional compound time-driven neuron copies and smaller incorporation time phases thus accomplishment of more accurate and corrects effects. Also, event-driven approaches are finest suitable for simulating modest neuron replicas with great intensities of the predictable values and accurateness.

\section{GPU SimUlations For STUdying BIOLOGICAL SySTEMS}

The huge demand of parallel computing and the need for more graphics performance in medical applications resulted in researchers exploring the use of high computing power inherent in GPUs. GPU performance is high when compared to CPU as it can differ by a factor of an order or more theoretically. Attaining high performance in the biological system modeling ideally requires parallel computation. There are some instances of achieving good results using a combination of CPU—GPU implementation [9]. Algorithms must be data parallel to perform efficiently on the GPUs. Also, algorithm execution should be in multiple threads with no clashing branches and using less memory than the total memory of the GPU.

The other factor that affects GPU performance is kernel complexity [15]. Biological systems modeling and simulation need large number of threads which is complementary to the acceleration of GPU. There are some factors like synchronization, memory usage that can reduce the speed of the serial execution. In recent times, use of frameworks like OpenCL and CUDA for ease of programming of GPUs is common [8]. Hybrid approach of using GPUs for massive data parallel portions and CPUs for fewer data parallel portions is promising. Use of hybrid approach introduces the problem of continuous sharing of the data by memory transmission above PCI definite bus. Major GPU manufacturers work to improve in this area and it still continues to be an area of concern. Reuse of GPU collections using normally used data structures and algorithms has started becoming a standard desirable practice.

Each biological system is complex and is inter-connected with many important physiological and pathological characters. The biological systems might be seen as difficult adaptive systems where each separate component is connected to complete various feedbacks and feed forward loops [10]. The non-linear relationships among these various factors can be interchanged among the components of the biological systems that petrify the study in biology at molecular and cellular stages which is extremely difficult otherwise $[14,16]$. It is not possible to achieve the minimum frame rate for simulating the biological systems using conventional computational schemes. The networks of numerous biochemical reactions can also be vie wed as biological systems. Such simulations require vast amount of floating point calculations. Simulation types and simulation methodologies using GPU are discussed in the rest of this section.

GPU power utilization has become a necessity when simulating biological systems. The primary reason for this is the enormous parallel threads and the graphics processing requirement of the complex biological environment needed. 
One use of GPU centered simulation is drug innovation. Drug discovery is the procedure by which different target medicines are exposed. Traditionally, medicines were exposed over recognizing the dynamic features from old-style medicines or by unexpected discovery [5].

Advanced chemical collections of artificial minor molecules, natural extracts were partitioned in integral cells or entire organisms to classify ingredients that require a necessary healing result in a procedure recognized as traditional pharmacology [16]. Meanwhile sequencing of human genome permits speedy copying and synthesis of huge amounts of cleaned proteins. It enables showing of large complex collections besides remote biological goals that are theorized to be illness revising in a procedure known as opposite pharmacology. Successes from these studies are then verified in cells then in animals for effectiveness [3].

In tissue simulation, GPUs are used in conjunction with CPUs to simulate various biochemical reactions involved in the tissue and with the other systems connected with the tissue. Simulation also helps to know the effects of stress and strain on tissue and how the tissue behaves with change in pressure. The material characteristics of many tissues have not yet been understood. GPU based tissue simulation helps in knowing the tissue properties without actually performing surgery. One of the applications of tissue simulation is modeling of the tissue based Computed Tomography (CT) image. The main objective here is to build a simulation procedure that plans the tissue surgical analysis using CT images collected from the patient [7].

Biological pathways contain several responding biochemical types and also responses. Simulation of these biological pathways except for the trivial cases is computationally rigorous due to difficulty in the schemes and their interactions [20]. These pathways reveal substantial difficulty in several important cellular procedures. Hence, there is a strong requirement for innovative primary simulation procedures and also for fresher calculating stages, systems and methods. HPP is used to simulate compound biological pathways [5].

Protein prediction function approaches are methods that bioinformatics investigators customize to allocate biological characters to proteins. These proteins are usually, ones poorly calculated or foreseen, centered on genome sequence statistics. These forecasts are frequently focused by statistics intensive computational techniques [10]. Examples are nucleic acid arrangement homology, gene expression profiles, the approximations to forecast the purpose of a protein is statistics driven computational procedure. The data is usually from nucleic acid series and gene expressions which are data intensive structures. GPU helps in simulating such data intensive simulations.

\section{CONCLUSION}

Modeling and simulation plays an important role in modern research whether it is pure or applied sciences. The complexity of biological systems, subsystems and interactions among them demand huge computing resources to simulate them. Advancements in heterogeneous parallel processing have helped in handling the complexities of simulating the biological systems by utilizing the multicore CPU and multi core GPU architecture. Heterogeneous parallel processing for the simulation of biological systems in medical applications involves developing algorithms, data structures and other tools to model biological systems and implementing them on heterogeneous parallel processing platforms. The paper reviews the problem areas in systems biology that have good potential for modeling and simulation using heterogeneous parallel processing platforms.

\section{REFERENCES}

[1] Youssef Chahibi, Sasitharan Balasubramaniam et.al., "Molecular Communication Modeling of Antibody-mediated Drug Delivery Systems", IEEE Transactions on Biomedical Engineering, vol. 62, no. 7, pp. 1683-1695, July 2015.

[2] I.F. Akyildiz et al., "Nanonetworks: A new communication paradigm at molecular level," Computer Networks, vol. 52, no. 12, pp. 2260-2279, Aug. 2008.

[3] G. Z. Ferl et al., "A predictive model of therapeutic monoclonal antibody dynamics and regulation by the neonatal FC receptor (FCRN)," Ann. Biomed. Eng., vol. 33, no. 11, pp. 1640-1652, 2005.

[4] A. Garg and J. P. Balthasar, "Physiologically-based pharmacokinetic (PBPK) model to predict IgG tissue kinetics in wild-type and FcRnknockout mice," J. Pharmacokinetics Pharmacodyn., vol. 34, no. 5, pp. 687-709, 2007.

[5] M. Pierobon and I. F. Akyildiz, "Diffusion-based noise analysis for molecular communication in nanonetworks," IEEE Trans. Signal Process, vol. 59, no. 6, pp. 2532-2547, Jun. 2011.

[6] M. Pierobon and I. F. Akyildiz, "Noise analysis in ligand-binding reception for molecular communication in nanonetworks," IEEE Trans. Signal Process, vol. 59, no. 9, pp. 4168-4182, Sep. 2011.

[7] Solli DR, Ropers C, Koonath P, and Jalali B, "Optical rogue waves", Nature 450, pp 1054-1057, 2007.

[8] Ng W, Rockwood T, and Reamon A, "Demonstration of ChannelStitched Photonic Time-Stretch Analog-to-Digital Converter with ENOB $\geq 8$ for a $10 \mathrm{GHz}$ Signal Bandwidth", GOMACTech-14, Charleston, South Carolina, 2014.

[9] Goda K, Tsia KK, and Jalali B, "Serial time-encoded amplified imaging for real time observation of fast dynamic phenomena", Nature 458, pp. 1145-1149, 2009.

[10] Zhang C, Xu Y, Wei X, Tsia KK and Wong KKY, "Time-stretch microscopy based on time-wavelength sequence reconstruction from wideband incoherent source", Appl. Phys. Lett. pp. 105, 2014.

[11] Mahjoubfar A, Goda K, Wang C, Fard A, Adam J, Gossett DR, Ayazi A, Sollier E, Malik O, Chen E, Liu Y, Brown R, Sarkhosh N, Di Carlo D and Jalali B, "3D ultrafast laser scanner", Proc. SPIE 8611: 86110N, 2013.

[12] Diebold ED, Buckley BW, Gossett DR and B. Jalali B, "Digitally synthesized beat frequency multiplexing for sub-millisecond fluorescence microscopy", Nature Photon. 7, pp. 806-810, 2013.

[13] Asghari MH and Jalali B, "Anamorphic transformation and its application to time bandwidth compression", Appl. Opt. 52, pp. 6735-6743, 2013.

[14] Asghari MH and Jalali B, "Experimental demonstration of realtime optical data compression", Appl. Phys. Lett. 104, pp. 1-4, 2014.

[15] Jalali B, Chan J and Asghari MH, "Time bandwidth engineering", Optical, pp 23-31, 2014.

[16] Valley GC, Sefler GA, and Shaw TJ, "Compressive sensing of sparse radio frequency signals using optical mixing", Optics letters 37, pp. 4675-4677, 2012.

[17] Haseong Kim and Erol Gelenbe, "Reconstruction of Large-Scale Gene Regulatory Networks Using Bayesian Model Averaging", 
IEEE TRANSACTIONS ON NANOBIOSCIENCE, vol. 11, no 3, pp. 1536-1241, Sep. 2012.

[18] M. Carro, W. Lim, M. Alvarez, R. Bollo, X. Zhao, E. Snyder, E. Sulman, S. Anne, F. Doetsch, and H. Colman, "The transcriptional network for mesenchymal transformation of brain tumours", Nature, , vol. 463, no. 7279, pp. 318-325, 2009.

[19] D. Erwin and E. Davidson, "The evolution of hierarchical gene regulatory networks", Nat. Rev. et., pp. 141-148, vol. 10, no. 2, 2009.

[20] E. Gelenbe, "A unified approach to the evaluation of a class of replacement algorithms", IEEE Trans. Comput., pp. 611-618, vol. C-100, no. 6, 1973.

[21] E. Gelenbe, "Steady-state solution of probabilistic gene regulatory networks", Phys. Rev. E, vol. 76, no. 3, pp. 031903, 2007.

[22] E. Gelenbe and G. Hebrail, "A probability model of uncertainty in data bases", in Proc. 2nd Int. Conf. Data Eng., 1986, pp. 328-333.

[23] Yong Zhang,peng Li, "Gene-Regulatory Memories: ElectricalEquivalent Modeling, Simulation and Parameter Identification", IEEE/ACM International Conference on Computer-Aided Design Digest of Technical Papers, 2009

[24] Gibson Hu et.al, "A Self-Organizing Nano Particle Simulator and Its Applications", Adaptive Systems Team, Autonomous Systems Laboratory ICT Centre, CSIRO, Australia. ASA/ESA Conference on Adaptive Hardware and Systems.

[25] Smith R. D., Dunbar J. B., Ung P. M. U., Esposito E. X., Yang C. Y., Wang S. M., Carlson, and H. A. CSAR, "Combined Evaluation Across All Submitted Scoring Functions", J. Chem. Inf. Model., vol. 51, pp. 2115-2131, 2011.
[26] Miller B. R., McGee T. D., Swails J. M., Homeyer N., Gohlke H., Roitberg A. E.,"MMPBSA., py: An Efficient Program for EndState Free Energy Calculations", J. Chem. Theory Computation., vol. 8, pp. 3314-3321, 2012.

[27] N. N. Petrishchev and I. A. Mikhailova, "Influence of some hydrodynamic factors of thrombus formation in microvessels", Microvasc. Res., pp 6-12, vol. 49, no. 1, 1995.

[28] Kirshner D. A., Nilmeier J. P., Lightstone F. C., "Catalytic site identification-a web server to identify catalytic site structural matches throughout PDB.”, Nucleic Acids Res. vol. 41, pp. W256-W265, 2013.

[29] Andre Gruning and Sander M. Bohte, "Spiking Neural Networks: Principles and Challenges", University of Surrey, United Kingdom CWI, Amsterdam, The Netherlands, ESANN 2014 proceedings, European Symposium on Artificial Neural Networks, Computational Intelligence and Machine Learning. Bruges (Belgium), 23-25 April 2014.

[30] Jesus A. et al, "Event and Time Driven Hybrid Simulation of Spiking Neural Networks", IWANN 2011, Part I, LNCS 6691, pp. 554561, 2011. c Springer, Verlag Berlin Heidelberg 2011.

[31] Luca Felicetti, Mauro Femminella and Gianluca Reali, "Simulating an in vitro experiment on nanoscale communications by using BiNS2", Nano Communication Networks, Elsevier, pp. 172-180, vol. 4, issue. 4, Dec 2013.

[32] S Kang, S Kahan, J McDermott, N Flann, I Shmulevich, "Biocellion: accelerating computer simulation of multicellular biological system models", Bioinformatics, Epub, Jul 26, 2014.

[33] Albert Lin, C.F. Chang, M.C. Lin and L.J Jan, "High-performance computing in remote sensing image compression", Proc SPIE 8183, High-Performance Computing in Remote Sensing, 81830C, Oct. 11, 2011. 